\title{
All order I.R. finite expansion for short distance behavior of massless theories perturbed by a relevant operator.
}

\author{
Riccardo Guida ${ }^{1}$ and Nicodemo Magnoli ${ }^{1,2}$ \\ ${ }^{1}$ Dipartimento di Fisica - Università di Genova \\ Via Dodecaneso, 33 - 16146 Genova, Italy \\ ${ }^{2}$ Istituto Nazionale di Fisica Nucleare-Sez. Genova \\ Via Dodecaneso, 33 - 16146 Genova, Italy \\ e-mail: guida@ge.infn.it, magnoli@ge.infn.it
}

\begin{abstract}
We consider here renormalizable theories without relevant couplings and present an I.R. consistent technique to study corrections to short distance behavior (Wilson O.P.E. coefficients) due to a relevant perturbation. Our method is the result of a complete reformulation of recent works on the field, and is characterized by a more orthodox treatment of U.V. divergences that allows for simpler formulae and consequently an explicit all order (regularization invariant) I.R. finitess proof. Underlying hypotheses are discussed in detail and found to be satisfied in conformal theories that constitute a natural field of application of this approach.
\end{abstract}

GEF-Th-12/1995

$11 / 1995$ 


\section{Introduction}

We present here a consistent technique to study the corrections to the short distance behavior of a renormalizable euclidean quantum field theory without relevant couplings (i.e. with positive mass dimension), when a relevant perturbation is introduced.

It is well known that expansions in relevant parameters around field theories without a mass scale are plagued by I.R. divergences. Considerable effort on how to deal with these singularities has been done, for generic super-renormalizable quantum field theories [1] as well as for some specific cases [2], but the present situation is such that any general and (especially) practical method that could face these I.R. divergences is always welcome.

The possibility of doing an I.R. finite expansion in a relevant parameter is particularly appealing in the case of (two dimensional) conformal field theories. After the first pioneering work [3], understanding on this subject has been greatly increased and many exact results have been found (see for instance [4]). In addition it soon became clear that those models can be taken as a starting point to get information on non conformally invariant theories [5], [6]. From a point of view of statistical mechanics, [7], conformal field theories are fixed points of the renormalization group and the line of research we are considering can be rephrased as "how to go outside the fixed point". We think that the technique introduced here finds a natural field of application within this framework because it gives a general way to describe the short distance behavior of a theory outside the fixed point, useful to test eventual exactly solved deformations but essential to give new light in all the other cases.

The method described in this paper is a reformulation of the independent works of Al.B. Zamolodchikov [8] and of Mikhak and Zarkesh [10] (that applied techniques developed by Sonoda [9]).

The first idea underlying the approach is present already in earlier papers of Wilson [11] and Wegner [12] (see also the more recent [13]): to deal with I.R. singularities is essential to redefine composite fields in such a way to confine the terms non analytic in the generalized masses (potentially present in all the interesting correlators) inside the V.E.V. of the composite fields, enforcing in this way the regularity of Wilson coefficients.

The second step is to give a formal expression of the derivatives of Wilson coefficients with respect to the generalized masses in terms of (generally unknown) correlators of the deformed theory: this has been realized by per-

turbative expansions in [8], and by using the "Variational Formulae" in 99 (a kind of Action Principle, see Section 1). 
The third step is to regularize the theory with an I.R. cutoff and to take the limit of zero generalized masses: clearly at this stage one has to face the potential I.R. divergences. The other main idea of the approach, [8, 9] (and [10 for an explicit proof up to second order), is that the convergence properties of O.P.E. should guarantee the cancellation of I.R. divergences. After keeping into account this fact the derivatives of the Wilson coefficients can be computed by evaluation of I.R. cutoff integrals involving exactly known correlators of the massless field theory.

Our present contribution is an improvement and an extension of the previously mentioned works, in order to give a simplified and U.V. consistent method with well defined applicability conditions that is I.R. finite at all orders.

First in Section 2 we state clearly which are the underlying, minimal hypotheses to be satisfied for the method to work.

Secondly, by using the orthodox renormalized Action Principle (that has a simpler form with respect to [9]), an explicit inductive proof of I.R. finitess and a detailed discussion of the implications of cancellation of I.R. divergences is given in Section 3. It is also shown that final expressions do not depend on the concrete form of the I.R. regularization (whose choice becomes a matter of convenience).

In Section 1 we treat the correct definition of renormalized composite operators to be chosen in such a way that Action Principle holds. We shall see that from this requirement arises the problem of existence of numerical constants (essentially present in the operators' V.E.V.) whose expression in terms of deformed theory correlators is well defined but in general uncomputable without additional information.

In Section 5.1 we compute the mass corrections to the short distance behavior of the propagator of two dimensional free euclidean fermions, while in Section 5.2 we study as a further example of our approach the mass corrections to the spin spin correlator in the Ising model.

\section{The general method and underlying hypotheses}

We present in this Section the ideas of the approach (see also [8, 9, 10]) and state a clear list of the underlying hypotheses (which are essentially three).

The goal is the reconstruction of the short distance behavior of correlation functions of composite operators of a $D$ dimensional euclidean field theory without dimensional couplings, perturbed by one or more operator 
$\mathcal{O}_{i}$ (of canonical dimension $x_{i}$ ) with dimensionful couplings $m^{i}$ ("generalized masses" of dimension $\left.y_{i} \equiv D-x_{i}\right)$ :

$$
\Delta S=-\int d x \sum_{i} m_{B}^{i} \mathcal{O}_{i B}(x)
$$

(notice the minus sign above, taken for convenience). We will refer to this model as "deformed theory" while the unperturbed one will be called "massless theory". The perturbation operator $\mathcal{O}_{i}$ is supposed here relevant $\left(0<x_{i}<D\right)$ to restrict the number of renormalization conditions needed to define its multiple insertions, see Section 4 .

We will assume that all correlators of the deformed and of the massless theory to be well defined.

The massless theory is supposed to be at least perturbatively U.V. renormalizable with respect to some dimensionless coupling $\lambda$; furthemore we will require that only logarithmic corrections to tree level scale invariance can arise.

The eventual perturbative (with respect to $\lambda$ ) knowledge of correlators at $m^{i}=0$ gives a perturbative estimation of each term of the mass expansions of the correlators of the deformed theory; nevertheless in the particular case of (two dimensional) conformal field theories (bare) correlators are exactly known and thus coefficients of mass expansion can be exactly computed. In the following we will forget the eventual dependence in $\lambda$ with the convention that all formulae should be intended to hold order by order in renormalized perturbative expansion with respect to $\lambda$ in the general case. Notice that the absence of super-renormalizable couplings guarantees that I.R. problems do not arise in massless theory if a renormalization scheme without additional unphysical masses is used (see e.g. discussion in the second and third papers of [16]).

The short distance behavior of the deformed theory is described by the Operator Product Expansion [11]:

$$
<\left[\Phi_{a_{1}}\left(r_{1}\right) \cdots \Phi_{a_{n}}\left(r_{n}\right)\right] X>_{m} \sim C_{a_{1} \cdots a_{n}}^{c}\left(r_{1}-r_{n}, \cdots r_{n-1}-r_{n} ; m\right)<\left[\Phi_{c}\left(r_{n}\right)\right] X>_{m},
$$

where $\Phi_{b}$ are a complete set of composite operators (that reduce in the limit $m^{i} \rightarrow 0$ to the operators of the massless theory), $X$ is a multilocal product of other fields localized "far away" from the $r_{i}$, the suffix $m$ refers to deformed theory, and the convergence of the series will be discussed below. In the following we will denote the renormalized multiple insertions of operators, see Section 1 , enclosing them with $[\cdots]$.

We will present an I.R. finite technique to compute multiple derivatives of Wilson coefficients $C_{a_{1} \cdots a_{n}}^{c}$ with respect to (renormalized) $m^{i}$ at the point 
$m^{i}=0$ in terms of integrated correlators of the massless theory. We will assume the following:

Hypothesis 1 (Regularity): an U.V. renormalization scheme for correlators of the deformed theory is assumed such that counterterms are polynomial in renormalized generalized masses (Minimal Mass Dependence, MMD). Furthermore, the Wilson coefficients are supposed to be regular (i.e. $\mathrm{C}^{\infty}$ ) in generalized masses at $m^{i}=0$.

Minimal Mass Dependence guarantees the smoothness of the $m^{i} \rightarrow 0$ limit from the point of view of U.V. renormalization, property which will be essential in the following. Any subtraction scheme that subtracts only the infinite part in the underlying regularization (Minimal Subtraction) is expected to satisfy MMD because no additional mass dependence is introduced in counterterms (apart from the trivial powerlike factors) that gives the correct physical dimension.

We remark that Wilson coefficients and Minimal Mass Dependence are not independent: at least in the framework of perturbative renormalization of quantum field theories MMD is equivalent to regularity of Wilson coefficients. This can be understood as follows: first we remind that Minimal Subtraction in Dimensional Renormalization (MS) satisfies MMD, [19], and regularity, [20]. Secondly any new scheme satisfying MMD will differ from MS by means of finite counterterms, polynomial in the masses. In particular the change of scheme for renormalized operators will amount to the transformation

$$
\left[\Phi_{a}\right]^{\prime}=N_{a}^{b}\left[\Phi_{b}\right]
$$

with $N_{a}^{b}=\delta_{a}^{b}+Y_{a}^{b}$ and $Y$ a matrix of order $O(\hbar)$ that is polynomial in the masses. Wilson coefficients will change accordingly as

$$
C_{a_{1} \cdots a_{n}}^{\prime c}=N_{a_{1}}^{b_{1}} \cdots N_{a_{n}}^{b_{n}} N_{d}^{-1}{ }_{d}^{c} C_{b_{1} \cdots b_{n}}^{d}
$$

and the new ones will be clearly regular in $m=0$ as the ones in MS scheme. Conversely in a scheme with non regular counterterms the matrix $N_{a}^{b}$ above will have some non regular entry and so for Wilson coefficients.

We want to emphasize furthermore that the stronger (nonperturbative) assumption of analyticity of Wilson coefficients in generalized masses would ensure the convergence of the Taylor series we are building up here, which is only asymptotic in the general case.

The second assumption necessary to compute derivatives with respect to generalized masses is:

Hypothesis 2 (Action Principle): it is assumed that for each renormalized generalized mass $m^{i}$ a conjugate operator $\mathcal{O}_{i}$ exists such that the 
derivative with respect to $m^{i}$ is realized as:

$$
\partial_{m^{i}}<[X]>_{m}=\int d x<\left[: \mathcal{O}_{i}(x): X\right]>_{m} \quad: \mathcal{O}_{i}: \equiv \mathcal{O}_{i}-<\left[\mathcal{O}_{i}\right]>_{m}
$$

for each multilocal operator $X$.

The Action Principle [14] is well known to hold in perturbative renormalization, in the classical B.P.H.Z. framework [15] as well as in other schemes satisfying Hypothesis 1 such as Dimensional Renormalization [16 and Analytic Renormalization [17] (see also [18] for Differential Renormalization). We will come back to this argument in Section 4 .

The simplicity of Eq.(2.5) compared to "Variational Formulae" [9] is the key of our approach that will allow us to deal with expressions for derivatives of generic order and to give the inductive IR finitess proof.

Let us finally have a glance at the method in a simple case, to introduce the final hypothesis. Suppose we want to compute the first derivative with respect to $m^{i}$ (in $m^{i}=0$ ) of the two point Wilson coefficient $C_{a b}^{1}$ (with $a, b, 1$ referring to renormalized operators $\left.\Phi_{a}, \Phi_{b}, 1\right)$. Let us consider the quantity (remainder of O.P.E.)

$$
\Delta^{(N)}\left(X_{R}, m\right) \equiv<\left[\left(\Phi_{a}(r) \Phi_{b}(0)-\sum_{c}^{x_{c} \leq N} C_{a b}^{c} \Phi_{c}(0)\right) X_{R}\right]>_{m}
$$

in which $x_{c}$ is the canonical dimension of operator $\Phi_{c}$ and $X_{R}$ is the trivial operator $\left(X_{R}=1\right)$ or is a multilocal operator with support in

$$
E_{R} \equiv\{x /|x|>R\}
$$

such that $R>|r|$. (In the following we also denote by $I_{R}$ the complement set of $E_{R}$, i.e. $\left.I_{R}=\mathbf{R}^{D}-E_{R}\right)$.

We then take derivatives of both sides with respect to $m^{i}$ : by using Action Principle we can write

$$
\begin{aligned}
\partial_{i} \Delta^{(N)}\left(X_{R}, m\right)= & \int d x<\left[: \mathcal{O}_{i}(x):\left(\Phi_{a} \Phi_{b}-\sum_{c}^{x_{c} \leq N} C_{a b}^{c} \Phi_{c}\right) X_{R}\right]>_{m} \\
& -\sum_{c}^{x_{c} \leq N} \partial_{i} C_{a b}^{c}<\left[\Phi_{c} X_{R}\right]>_{m} .
\end{aligned}
$$

It is evident that the in the limit of zero generalized masses the integral in the right hand side might be I.R. divergent. To deal with this problem we must proceed as follows, [9]. First we split the integral in two pieces,

$$
\partial_{i} \Delta^{(N)}\left(X_{R}, m\right)=\int_{I_{R_{1}}} d x<\left[: \mathcal{O}_{i}:\left(\Phi_{a} \Phi_{b}-\sum_{c}^{x_{c} \leq N} C_{a b}^{c} \Phi_{c}\right) X_{R}\right]>_{m}
$$




$$
\begin{aligned}
+ & \int_{E_{R_{1}}} d x<\left[: \mathcal{O}_{i}:\left(\Phi_{a} \Phi_{b}-\sum_{c}^{x_{c} \leq N} C_{a b}^{c} \Phi_{c}\right) X_{R}\right]>_{m} \\
& -\sum_{c}^{x_{c} \leq N} \partial_{i} C_{a b}^{c}<\left[\Phi_{c} X_{R}\right]>_{m},
\end{aligned}
$$

in which $R_{1}>R$. Then we observe that the second integral can be rewritten as

$$
\int_{E_{R_{1}}} d x<\left[: \mathcal{O}_{i}:\left(\Phi_{a} \Phi_{b}-\sum_{c}^{x_{c} \leq N} C_{a b}^{c} \Phi_{c}\right) X_{R}\right]>_{m}=\Delta^{(N)}\left(X_{R}^{\prime}, m\right)
$$

i.e. in the same form of Eq. (2.6) with $X_{R}^{\prime} \equiv \int_{E_{R_{1}}}: \mathcal{O}_{i}: X_{R}$ satisfying the same hypothesis on the support as $X_{R}$.

If we could assume the (weak) convergence of O.P.E. series when inserted in correlation functions containing "far" operators $X_{R}$ for the deformed theory, we would say that the limit $N \rightarrow \infty$ of $\Delta^{(N)}\left(X_{R}, m\right)$ of $(2.6)$ is zero together with all its mass derivatives and in particular the $N \rightarrow \infty$ limit of Eq.(2.10) would be zero for arbitrary $R_{1}>R>|r|$ : the subsequent limit $m \rightarrow 0$ of Eq.(2.9) would be safe and could be exchanged with the first (I.R. cut off) integral with the desired result

$$
\begin{aligned}
0= & \lim _{N \rightarrow \infty}\left\{\int_{I_{R_{1}}} d x<\left[: \mathcal{O}_{i}:\left(\Phi_{a} \Phi_{b}-\sum_{c}^{x_{c} \leq N} C_{a b}^{c} \Phi_{c}\right) X_{R}\right]>_{m=0}\right. \\
& \left.-\sum_{c}^{x_{c} \leq N} \partial_{i} C_{a b}^{c}<\left[\Phi_{c} X_{R}\right]>_{m=0}\right\}
\end{aligned}
$$

(the suffix $<>_{m=0}$ will be omitted in the following).

We think that, in spite of some argument in favor 21], convergence of O.P.E. is far from being reasonably proved in a quantum field theory with (generalized) mass; in particular the existence of terms of the form $e^{-1 /\left(m r^{y}\right)}$ could spoil the convergence of the expansion for small $r$ (see the first paper in [7]).

Nevertheless for our purposes it suffices the following weaker hypothesis that we state here for general Wilson coefficients.

Hypothesis 3 (O.P.E. asymptotic weak convergence): it is assumed that the remainder of O.P.E.

$$
\Delta^{(N)}\left(X_{R}, m\right) \equiv<\left[\left(\Phi_{a_{1}}\left(r_{1}\right) \cdots \Phi_{a_{n}}\left(r_{n}\right)-\sum_{c}^{x_{c} \leq N} C_{a_{1} \cdots a_{n}}^{c} \Phi_{c}\right) X_{R}\right]>_{m}
$$


$\left(X_{R}\right.$ being the unity or an arbitrary multilocal operator with support outside $\left.R>\left|r_{1}\right|, \cdots\left|r_{n}\right|\right)$ satisfies

$$
\lim _{N \rightarrow \infty} \lim _{m \rightarrow 0} \lim _{R \rightarrow \infty} \partial_{i_{1}} \cdots \partial_{i_{k}} \Delta^{(N)}\left(X_{R}, m\right)=0
$$

for each $k$. Equivalently we can write that

$$
\lim _{N \rightarrow \infty} \lim _{R \rightarrow \infty}<\left[\left(\Phi_{a_{1}}\left(r_{1}\right) \cdots \Phi_{a_{n}}\left(r_{n}\right)-\sum_{c}^{x_{c} \leq N} C_{a_{1} \cdots a_{n}}^{c} \Phi_{c}\right) X_{R}\right]>\sim 0
$$

in the sense of asymptotic series (in $m^{i}$ ).

First of all we notice that assumption Eq.(2.14) for $k=0$ requires the convergence of the O.P.E. in the underlying $m^{i}=0$ theory when inserted in correlators with "far" operators. This property is well known to hold in conformal field theories, [22]. Also from axiomatic [23] as well from perturbative 24] considerations we can say that in general quantum field theories O.P.E. is an asymptotic expansion in powers of $r$. But in the limit $R \rightarrow \infty$, by clustering, the contribution of $X_{R}$ in the correlator factorizes and the only physical scale in the O.P.E. becomes the generalized mass (the renormalization point $\mu$ cancels between V.E.V. and Wilson coefficients by Renormalisation Group invariance of the sum) and a remainder of order $O\left(r^{\nu}\right)$ is actually of the form $O\left(\left(m^{\frac{1}{y}} r\right)^{\nu}\right)$ by dimensional analysis, and thus the O.P.E series with respect to powers of the generalized masses reasonably becomes asymptotic.

Let us come back to our previous example. In the limit of Eq.(2.13) it is clear that the contribution from Eq.(2.10) vanishes. Observing that also $\partial_{i} \Delta$ vanishes, and choosing $X_{R}=1$ we obtain the formula

$0=\lim _{N, R_{1} \rightarrow \infty} \int_{I_{R_{1}}}<\left[: \mathcal{O}_{i}:\left(\Phi_{a} \Phi_{b}-\sum_{c}^{x_{c} \leq N} C_{a b}^{c}(0) \Phi_{c}\right)\right]>-\sum_{c}^{x_{c} \leq N} \partial_{i} C_{a b}^{c}(0)<\left[\Phi_{c}\right]>$

in which the limit over $m$ and $R$ were exchanged without problems for the surviving I.R. regulated quantities. (Notice also that (0) in Wilson coefficients refers to $m=0$; we will omit this specification for simplicity of notations in next Sections, being clear from the context if we are dealing with zero mass Wilson coefficients or not.)

To have a useful formula we must observe that in general, by dimensional considerations,

$$
\lim _{R_{i} \rightarrow \infty} \int_{I_{R_{1}}} d x_{1} \cdots \int_{I_{R_{k}}} d x_{k}<\left[: \mathcal{O}_{i_{1}}: \cdots: \mathcal{O}_{i_{k}}: \Phi_{c}(0)\right]>=0
$$

\footnotetext{
${ }^{1}$ In the rest of the paper we will exchange derivatives with respect to generalized masses with the limit over I.R. cutoff when the limit over $m$ follows, because at $m \neq 0$ the limit over $R$ is smooth.
} 
if $x_{c}-\sum_{j=1}^{k} y_{j}>0\left(y_{i}\right.$ being mass dimensions of $\left.m^{i}\right)$ due to the absence of a physical scale in the $m=0$ theory (we are using the fact that renormalization point $\mu$ does not give powerlike corrections).

By Eq.(2.16) the series in $c$ index is truncated and we can write

$$
\partial_{i} C_{a b}^{1}(0)=\lim _{R \rightarrow \infty} \int_{I_{R_{1}}} d x<\left[: \mathcal{O}_{i}:\left(\Phi_{a} \Phi_{b}-\sum_{c}^{x_{c} \leq y_{i}} C_{a b}^{c}(0) \Phi_{c}\right)\right]>.
$$

The goal of expressing the (first) derivative of a Wilson coefficient in term of well defined and known quantities of massless theory is then reached! In the next Section we will deal with higher order derivatives. Notice that all formulae that we will derive do not depend on the U.V. renormalization scheme, provided that Hypotheses 1-3 are satisfied. The choice of the scheme is a matter of taste and computational convenience.

\section{All order formulae and I.R. finitess proof}

In this Section we will give I.R. safe expressions for the $n^{\text {th }}$ derivative of the Wilson coefficients with respect to generalized masses by using the assumptions of previous Section. Then some consequences of these identities will be discussed.

We will prove by induction on $n$ the general form of $n^{\text {th }}$ derivative of (two operators) Wilson coefficient at zero masses and its I.R. finitess. Generalization to higher operator Wilson coefficients is straightforward.

We want to prove that at order $n$ the following relation holds for the deformed theory:

$$
\begin{aligned}
& \lim _{N \rightarrow \infty} \lim _{R \rightarrow \infty} \partial_{i_{1}} \cdots \partial_{i_{n}} \Delta^{(N)}\left(X_{R}, m\right) \sim \\
& \lim _{N, R \rightarrow \infty}\left\{\int_{I_{1}} d x_{1} \cdots \int_{I_{n}} d x_{n}<\left[: \mathcal{O}_{i_{n}}: \cdots: \mathcal{O}_{i_{1}}:\left(\Phi_{a_{1}} \Phi_{a_{2}}-\sum^{x_{b} \leq N} C_{a_{1} a_{2}}^{b} \Phi_{b}\right) X_{R}\right]>_{m}\right. \\
& -\sum^{x_{b} \leq N} \partial_{i_{1}} C_{a_{1} a_{2}}^{b} \int_{I_{2}} d x_{2} \cdots \int_{I_{n}} d x_{n}<\left[: \mathcal{O}_{i_{n}}: \cdots: \mathcal{O}_{i_{2}}: \Phi_{b} X_{R}\right]>_{m}+\mathrm{p} . \\
& -\sum^{x_{b} \leq N} \partial_{i_{1}} \partial_{i_{2}} C_{a_{1} a_{2}}^{b} \int_{I_{3}} d x_{3} \cdots \int_{I_{n}} d x_{n}<\left[: \mathcal{O}_{i_{n}}: \cdots: \mathcal{O}_{i_{3}}: \Phi_{b} X_{R}\right]>_{m}+\mathrm{p} . \\
& \left.\cdots-\sum^{x_{b} \leq N} \partial_{i_{1}} \cdots \partial_{i_{n}} C_{a_{1} a_{2}}^{b}<\left[\Phi_{b} X_{R}\right]>_{m}\right\}
\end{aligned}
$$

in which $I_{i} \equiv I_{R_{i}}$ for shortness and $R_{n}, R_{n-1} \cdots R_{1}>R>|r|$. The series are to be intended asymptotic as explained in the previous Section and with $p$. 
we mean all terms obtained interchanging the suffix $(1, \cdots, k-1)$ of Wilson coefficients' derivatives with $(k, \cdots, n)$ inside integrals without distinguishing the ordering. We observe that the left hand side of the previous expression is zero asymptotically due to Hypothesis 3, Eq.(2.14), and that due to the presence of I.R. cutoff the massless limit is I.R. safe as we shall use below.

It is clear by Hypothesis 3 that at $n=0$ Eq.(3.1) holds. Now let us assume that Eq.(3.1) holds at order $n$ and show that it holds at order $n+1$. Let us take derivative with respect to $m^{i_{n+1}}$ of both sides of Eq.(3.1) (and commute derivative with respect to limit over $R$, see footnote 1).

The action of $\partial_{i_{n+1}}$ on the correlators gives terms of the form

$$
\lim _{N, R \rightarrow \infty} \int d x_{n+1}<\left[: \mathcal{O}_{i_{n+1}}\left(x_{n+1}\right):\{n\}\right]>_{m}
$$

i.e. the same terms of order $n$ (contracted with Wilson coefficients as in (3.1)), indicated above with $\{n\}$, but with the additional insertion of : $\mathcal{O}_{i_{n+1}}:$, and terms in which derivative acts on Wilson coefficients present at order $n$. But by Eq.(3.1) (with $X_{R} \rightarrow \int_{E_{n+1}} d x_{n+1}: \mathcal{O}_{i_{n+1}}: X_{R}$ ) we obtain

$$
\lim _{N, R \rightarrow \infty} \int d x_{n+1}<\left[: \mathcal{O}_{i_{n+1}}:\{n\}\right]>_{m}=\lim _{N, R \rightarrow \infty} \int_{I_{n+1}} d x_{n+1}<\left[: \mathcal{O}_{i_{n+1}}:\{n\}\right]>_{m}
$$

if we restrict to $R_{n}, R_{n-1}, \cdots, R_{1} \geq R \rightarrow \infty$.

It is easy to check that the sum of the two contributions simply reproduces the $n+1$ expression according to (3.1) so that induction works Q.E.D.

If we choose $X_{R}=1$ in Eq.(3.1), take the $m^{i} \rightarrow 0$ limit and remind the dimensional selection rule Eq.(2.16) we obtain finally that, in the limit $R_{n}, R_{n-1}, \cdots, R_{1} \geq R \rightarrow \infty$, the series in index $b$ are actually finite sums and that

$$
\begin{aligned}
& \partial_{i_{1}} \cdots \partial_{i_{n}} C_{a_{1} a_{2}}^{1}= \\
& \lim _{R \rightarrow \infty}\left\{\int_{I_{1}} d x_{1} \cdots \int_{I_{n}} d x_{n}<\left[: \mathcal{O}_{i_{n}}: \cdots: \mathcal{O}_{i_{1}}:\left(\Phi_{a_{1}} \Phi_{a_{2}}-\sum_{b}^{*} C_{a_{1} a_{2}}^{b} \Phi_{b}\right)\right]>\right. \\
& -\sum_{b}^{*} \partial_{i_{1}} C_{a_{1} a_{2}}^{b} \int_{I_{2}} d x_{2} \cdots \int_{I_{n}} d x_{n}<\left[: \mathcal{O}_{i_{n}}: \cdots: \mathcal{O}_{i_{2}}: \Phi_{b}\right]>+\mathrm{p} \\
& \ldots \\
& \left.-\sum_{b}^{*} \partial_{i_{1}} \cdots \partial_{i_{n-1}} C_{a_{1} a_{2}}^{b} \int_{I_{n}} d x_{n}<\left[: \mathcal{O}_{i_{n}}: \Phi_{b}\right]>+\mathrm{p} .\right\}
\end{aligned}
$$

where $\sum_{b}^{*}<\left[: \mathcal{O}_{i_{n}}: \cdots: \mathcal{O}_{i_{k}}: \Phi_{b}\right]>$ is restricted to $\Phi_{b}$ such $x_{b} \leq y_{i_{k}} \cdots+y_{i_{n}}$. 
More generally if we keep in Eq.(3.1) a nontrivial $X_{R}$ and take the $m^{i} \rightarrow 0$ limit, we can observe that

$$
\int_{I_{k}} \cdots \int_{I_{n}}<\left[: \mathcal{O}_{i_{n}}: \cdots: \mathcal{O}_{i_{k}}: \Phi_{b}(0) X_{R}\right]>=O\left(R^{\delta}\right)
$$

in which we defined the I.R. degree of divergence of the correlator as

$$
\delta \equiv \sum_{j=k}^{n} y_{i_{j}}-x_{b}-x_{X_{R}}
$$

by simple dimensional considerations. It follows that all correlators with $\delta<0$ give vanishing contribution in the $R \rightarrow \infty$ limit and the series in the index $b$ are effectively truncated.

We obtain in all generality:

$$
\begin{aligned}
0= & \lim _{R \rightarrow \infty}\left\{\int_{I_{1}} d x_{1} \cdots \int_{I_{n}} d x_{n}<\left[: \mathcal{O}_{i_{n}}: \cdots: \mathcal{O}_{i_{1}}:\left(\Phi_{a_{1}} \Phi_{a_{2}}-\sum_{b}^{*} C_{a_{1} a_{2}}^{b} \Phi_{b}\right) X_{R}\right]>\right. \\
& -\sum_{b}^{*} \partial_{i_{1}} C_{a_{1} a_{2}}^{b} \int_{I_{2}} d x_{2} \cdots \int_{I_{n}} d x_{n}<\left[: \mathcal{O}_{i_{n}}: \cdots: \mathcal{O}_{i_{2}}: \Phi_{b} X_{R}\right]>+\mathrm{p} . \\
& \ldots \\
& -\sum_{b}^{*} \partial_{i_{1}} \cdots \partial_{i_{n-1}} C_{a_{1} a_{2}}^{b} \int_{I_{n}} d x_{n}<\left[: \mathcal{O}_{i_{n}}: \Phi_{b} X_{R}\right]>+\mathrm{p} . \\
& \left.-\sum_{b}^{*} \partial_{i_{1}} \cdots \partial_{i_{n}} C_{a_{1} a_{2}}^{b}<\left[\Phi_{b} X_{R}\right]>\right\}
\end{aligned}
$$

where $\sum_{b}^{*}<\left[: \mathcal{O}_{i_{n}}: \cdots: \mathcal{O}_{i_{k}}: \Phi_{b} X_{R}\right]>$ is restricted to $\Phi_{b}$ such $x_{b} \leq$ $y_{i_{k}} \cdots+y_{i_{n}}-x_{X_{R}}$.

Eq. 3.7) gives an infinite number of linear algebraic relations (one for each choice of $X_{R}$ ) among derivatives of Wilson coefficients up to order $n$. Of course consistency of the method (guaranteed by our assumptions) enforces that only a finite number of relations are independent and the system is not overconstrained.

Moreover it is important to observe that in each equation such as (3.7) the I.R. divergences of the same form cancel independently. In particular this

\footnotetext{
${ }^{2}$ As an example, in conformal field theories the particular choice $X_{R}=\Phi_{c}(z, \bar{z}) z^{\Delta} \bar{z}^{\bar{\Delta}}$ (with $z, \bar{z}$ complex coordinates of modulus $R$ and $\Delta, \bar{\Delta}$ the relative canonical dimensions of operator $\Phi_{c}$ in the underlying conformal field theory) will select $\partial_{i_{1}} \cdots \partial_{i_{n}} C_{a_{1} a_{2}}^{c}$ in the last term of Eq.(3.7), by orthonormality property of conformal operators.
} 
happens for terms of the same dimensionality (I.R. degree of divergence): if for fixed $a_{1}, a_{2}$ we define the finite set

$$
S_{\sigma} \equiv\left\{\partial_{i_{1}} \cdots \partial_{i_{k}} C_{a_{1}, a_{2}}^{b} / \sigma=\sum_{j=1}^{k} y_{i_{j}}+x_{b}\right\}
$$

it is clear that for every choice of $i_{1}, \cdots, i_{n}$ and $X_{R}$ in Eq.(3.7) we have linear relations only between elements of the same sets $S_{\sigma}$, because $\delta=$ $\sum_{j=1}^{n} y_{i_{j}}-x_{X_{R}}-\sigma$ for each term of (3.7).

At this point to compute a derivative of a Wilson coefficient belonging to a set $S_{\sigma}$ one has only to choose a convenient number of subrelations of (3.7) (varying $X_{R}$ or $n$ ) and solve a linear system. Notice that due to possible different form of I.R. divergences with the same dimensionality the number of relations obtained by I.R. cancellation might become even bigger. This can happen also when some quantum numbers are conserved.

We want to show now that, while the particular I.R. spatial cutoff we used was essential to derive Eq.(3.1) by induction, our final results, Eq.(3.4) and Eq.(3.7) hold for a wide class of I.R. regularizations. The point is that in Eq.(3.1) all the terms have the form (keeping only essential features)

$$
\lim _{m \rightarrow 0} \lim _{R_{i} \geq R \rightarrow \infty} \int d x_{1} \cdots \int d x_{k} \theta_{R_{1}}\left(x_{1}\right) \cdots \theta_{R_{k}}\left(x_{k}\right)<\cdots X_{R}>
$$

where $\theta_{R}(x)=\theta(|x|-R)$ (with $\theta$ the usual step function). Let us now consider a generic I.R. regulator function $\Theta_{R^{\prime}}(x)\left(R^{\prime}\right.$ being a new length parameter) such that $\lim _{R^{\prime} \rightarrow \infty} \Theta_{R^{\prime}}(x)=1$. Inserting 1 in the integrals and exchanging integration with these limits we have

$$
\lim _{m \rightarrow 0} \lim _{R_{i} \geq R \rightarrow \infty} \lim _{R_{i}^{\prime} \rightarrow \infty} \int d x_{1} \cdots \int d x_{k} \theta_{R_{1}}\left(x_{1}\right) \cdots \theta_{R_{k}}\left(x_{k}\right) \Theta_{R_{1}^{\prime}}\left(x_{1}\right) \cdots \Theta_{R_{k}^{\prime}}\left(x_{k}\right)<\cdots X_{R}>.
$$

It is clear that at $m \neq 0$ (the limit on $m$ being external) we can exchange limits over $R, R_{i}$ with those over $R^{\prime}$ and subsequently the limit over $R$ with integrations, obtaining

$$
\lim _{m \rightarrow 0} \lim _{R_{i}^{\prime} \rightarrow \infty} \int d x_{1} \cdots \int d x_{k} \Theta_{R_{1}^{\prime}}\left(x_{1}\right) \cdots \Theta_{R_{k}^{\prime}}\left(x_{k}\right)<\cdots X_{\infty}>.
$$

Proceeding exactly as done previously (exchanging limit over masses and over $R^{\prime}$, using selection rules at $m^{i}=0$ ) we obtain for a generic I.R. cutoff function $\Theta_{R}$ :

$$
0=\lim _{R_{i} \rightarrow \infty}\left\{\int d x_{1} \cdots \int d x_{n} \Theta_{R_{1}}\left(x_{1}\right) \cdots \Theta_{R_{n}}\left(x_{n}\right) \times\right.
$$




$$
\begin{aligned}
& <\left[: \mathcal{O}_{i_{n}}: \cdots: \mathcal{O}_{i_{1}}:\left(\Phi_{a_{1}} \Phi_{a_{2}}-\sum_{b}^{*} C_{a_{1} a_{2}}^{b} \Phi_{b}\right) X_{\infty}\right]> \\
- & \sum_{b}^{*} \partial_{i_{1}} C_{a_{1} a_{2}}^{b} \int d x_{2} \cdots \int d x_{n} \Theta_{R_{2}}\left(x_{2}\right) \cdots \Theta_{R_{n}}\left(x_{n}\right) \times \\
& <\left[: \mathcal{O}_{i_{n}}: \cdots: \mathcal{O}_{i_{2}}: \Phi_{b} X_{\infty}\right]>+\mathrm{p} .+\cdots \\
& -\sum_{b}^{*} \partial_{i_{1}} \cdots \partial_{i_{n-1}} C_{a_{1} a_{2}}^{b} \int d x_{n} \Theta_{R_{n}}\left(x_{n}\right)<\left[: \mathcal{O}_{i_{n}}: \Phi_{b} X_{\infty}\right]>+\mathrm{p} . \\
& \left.-\sum_{b}^{*} \partial_{i_{1}} \cdots \partial_{i_{n}} C_{a_{1} a_{2}}^{b}<\left[\Phi_{b} X_{\infty}\right]>\right\}
\end{aligned}
$$

(same meaning of the ${ }^{*}$ as before). It is clear that a clever choice of I.R. cutoff can considerably simplify computations. For instance it is easy to prove that when $X_{\infty}=1$ and the cutoff function $\Theta_{R}$ is rotation invariant, only scalar operators are involved in the expressions. This property will be used in Section 5

We close the Section noting that, while Eqs.(3.4), (3.7), were derived by using only of the condition $R_{n}, R_{n-1}, \cdots, R_{1} \geq R \rightarrow \infty$, with the result of having symmetric formulae, for practical calculations it is better [9] to force the I.R. cutoff to go to infinity in a hierarchical way, say, $R_{n}>>R_{n-1}, \cdots>>$ $R_{1} \geq R \rightarrow \infty$ so that we can perform first the integral with bigger cutoff $R_{n}$, neglecting subleading terms of order $O\left(\frac{R_{n-1}}{R_{n}}\right)$ and so on. This could not be the most convenient way to compute integrals in other I.R. regularizations: for instance in the case $\Theta_{Q_{i}}(x)=e^{i Q_{i} x}$ it is simpler to take all $Q_{i}=Q \rightarrow 0$.

\section{U.V. definition of composite operators}

In this Section we schematically review some known facts on the treatment of renormalized multiple insertions of composite operators with particular reference to the operator $\mathcal{O}_{m}$ conjugate to the generalized mass $m$ by the Action Principle Eq.(2.5) (we will deal here only with one generalized mass; extension to general case should be straightforward for the reader).

The foundations of renormalization are locality and power counting ([25], see 26] for a pedagogical introduction). Each composite operator $\Phi_{a}$ has a related power counting dimension equal to the canonical one $x_{a}$, (we will not consider oversubtracted operators here). Each insertion of $\Phi_{a}$ in a truncated connected one particle irreducible graph $\gamma$ changes the U.V. superficial degree of divergence $\omega_{\gamma}$ by the amount $\delta \omega=-y_{a}=-D+x_{a}$, generating

new divergences. Divergences related to one operator insertion are treated 
allowing for a mixing of each bare operator with those of lower power counting dimension to get a finite renormalized one, $\left[\Phi_{a}\right]$. Renormalized multiple insertions of composite operators (denoted here by $[\cdots]$ ) contain new divergences that should be cured by counterterms proportional to delta function and its derivatives, the so called contact terms: for instance

$$
\left[\Phi_{a}(x) \Phi_{b}(0)\right]=\left[\Phi_{a}(x)\right]\left[\Phi_{b}(0)\right]+\text { contact terms. }
$$

For detailed treatment of arbitrary multiple insertions see [27] and references therein. A practical way to deal with multiple insertions is obtained introducing sources $\omega^{a}(x)$ of dimension $y_{a}$ for composite operators $\left[\Phi_{a}\right]$, and allowing for counterterms proportional to monomial in the sources, in the composite fields and their derivatives of total dimension less then or equal $D$ (see e.g. [28, 29]).

For a composite operator to be well defined a normalization condition should be given in correspondence of each infinite subtraction. A renormalization scheme is a consistent and physical way to subtract divergences and to fix finite counterterms with explicit or implicit normalization conditions.

Equivalently, a renormalization scheme can be seen as a consistent technique to build up well defined tempered distributions (satisfying physical requirements, 25) from "bare expressions" that do not define a distribution and to fix finite counterterms arising from the ambiguities of this extension. This point of view is more suitable for conformal field theory where "bare" exact expressions are known for correlators of composite operators at different positions.

For instance the bare correlator with two insertions of the operator $\mathcal{E} \equiv$ - : $\bar{\psi} \psi:$ in the free massless $D=2$ euclidean Dirac Fermions model (that is a conformal field theory) is

$$
<\mathcal{E}(r) \mathcal{E}(0)>_{B}=\frac{1}{2 \pi^{2}} \frac{1}{|r|^{2}}
$$

but $1 / r^{2}$ is not a distribution. This can be understood because it is not locally integrable with respect to the measure $d^{2} r$. A distribution $T(r)$ that extends the bare correlator can be defined (in polar coordinates) as the solution of the distributional equation

$$
|r| T(r)=\frac{1}{2 \pi|r|}
$$

$(1 / r$ is a distribution) whose general solution will have the form

$$
T(|r|)=T_{P}(|r|)+T_{H}(|r|)
$$


where $T_{P}$ is a particular solution of the complete equation such as $F p \frac{1}{2 \pi|r|^{2}}$ (Hadamard finite part, see Appendix B) and $T_{H}=C_{0} \delta(|r|)$ is a solution of the homogeneous equation. The renormalized correlators will be equal to $T$ and the dimensionless finite contact term $C_{0}$ will be fixed after imposing a normalization condition. The alternative definition

$$
|r|^{n} T(r)=\frac{1}{2 \pi}|r|^{n-1}
$$

for some $n>1$ would give a $T_{H}=\sum_{k=0}^{n-1} C_{k} \delta^{(k)}(|r|)$ and would change drastically the short distance behavior of the correlator: in this sense is not physically relevant.

From our discussion it appears clearly that only a finite number of insertions of a relevant operator give U.V. divergences (and contact terms to be eventually fixed): this is the main practical motivation to restrict to relevant perturbations, while all formulae presented in Section 3 can be extended formally to any marginal operator (at any finite order).

After this brief digression let us come to the problem of defining renormalized operators (i.e. of fixing schemes) satisfying the Action Principle. The point is that, while being clearly true at bare level (think about functional integration), the Action Principle might be not satisfied in a particular scheme, being possibly violated by renormalization. In this case the starting point scheme must be corrected (i.e. changed) by finite counterterms to enforce AP. It follows in our case that all the U.V. divergent insertions of the renormalized composite operator $\mathcal{O}_{m}$ to be cured in a chosen scheme might require additional contact term to be fixed in such a way that Eq.(2.5) holds. These contact terms result to be finite numbers (apart the trivial power of $m$ ) whose expression in terms of integrals of U.V. renormalized correlators (in the original scheme) is known and the only free parameter characterizing the composite operators is the usual renormalization point $\mu$.

Of course it is possible that in some renormalization scheme the Action Principle holds automatically without finite renormalization; this is the case of Dimensional Renormalization, [16] where no additional contact terms are needed and life is easier.

In our approach we are interested only in the zero mass limit, thus all dimensionful contact terms vanish and are not involved in our expressions. Only dimensionless contact terms could survive in zero mass correlators: they have a definite expression in terms of massive integrals but cannot be computed in terms of the massless quantities only. This situation arises when one tries to impose the condition

$$
\partial_{m}^{k}<\left[\Phi_{a}\right]>_{m}=\int d x_{1} \cdots d x_{k}<\left[\mathcal{O}_{m}\left(x_{1}\right) \cdots \mathcal{O}_{m}\left(x_{k}\right) \Phi_{a}\right]>_{m}
$$


in the case when $x_{a}=k y_{m}$ (in general when the I.R. degree of divergence $\delta$ defined in Eq.(3.6) is zero). Equation (4.6) presents non trivial U.V. renormalization and also is I.R. singular at $m=0(\log m$ terms are clearly present in the operator V.E.V.): it follows that a numerical constant that parameterizes the I.R. behavior of the operator $\Phi_{a}$ in the deformed theory is left not fixed (but expressed in terms of deformed theory correlators).

These unknown constants will parameterize our results. In the case of one generalized mass we can always confine this ambiguity to the composite operators V.E.V., as will be shown in the next Section with two examples. See also the related paper 30]

Before closing this Section we comment here that "Variational Formulae" 3 of Ref. [9] were invented with the goal of building up a geometrization of the theory space of the renormalized theories, [31, with a connection expressed in terms of a set of finite arbitrary "contact terms". We feel that the idea of geometrization (interesting by itself) is somewhat incompatible with straightforward calculations and in our pragmatic approach we will try to minimize the number of contact terms. In particular a locally flat coordinate system (no connection at all) is obtained when schemes with a built in Action Principle can be used.

\section{Two examples}

\subsection{A toy model example: short distance behavior of two dimensional free fermion propagator}

In this subsection we compute the mass corrections to the short distance behavior of the two dimensional euclidean free Dirac fermions propagator up to order $m^{2}$ by using the knowledge of the massless theory. We will use as renormalization scheme the Minimal Subtraction (MS) in Dimensional Regolarization.

To fix notations (we use that of [28]) we write here the exact expression

\footnotetext{
${ }^{3}$ In our mind "Variational Formulae" are incomplete because do not give a general definite prescription to build up distributions from bare correlators (i.e. do not define a scheme). In particular it seems to us that there is no prescription to subtract singularities arising when three or more operators collapse to a point, as happens at high derivative orders.
} 
for the propagator we want to recover:

$$
<\psi(r) \bar{\psi}(0)>_{m}=-\frac{m}{2 \pi} K_{0}(m|r|)-\frac{m}{2 \pi} \frac{\hat{r}}{|r|} K_{1}(m|r|)=\int \frac{d^{2} p}{(2 \pi)^{2}} e^{-i p x} \frac{1}{i \hat{p}-m}
$$

in which $\hat{r}=r^{\mu} \sigma^{\mu}, \mu=1,2$.

We want to use Eq.(3.4) with $n=1,2$, that we rewrite here (neglecting the trivially zero contributions and changing slightly notations):

$$
\begin{aligned}
& \partial_{m} C^{1}(r)=\lim _{R_{1} \rightarrow \infty}\left\{\int^{R_{1}} d^{2} x_{1}<\left[: \mathcal{O}_{m}\left(x_{1}\right):\left(\psi(r) \bar{\psi}(0)-C^{\mathcal{O}_{m}} \mathcal{O}_{m}\right)\right]>\right\} \\
& \partial_{m}^{2} C^{1}(r)=\lim _{R_{2}>>R_{1} \rightarrow \infty}\left\{\int^{R_{1}} d^{2} x_{1} \int^{R_{2}} d^{2} x_{2}<\left[: \mathcal{O}_{m}\left(x_{1}\right):: \mathcal{O}_{m}\left(x_{2}\right):(\psi(r) \bar{\psi}(0)\right.\right. \\
& \left.\left.-C^{1}-C^{\Phi_{2}^{c}} \Phi_{2}^{c}\right)\right]>-\int^{R_{1}} d^{2} x_{1} \partial_{m} C^{\mathcal{O}_{m}}<\left[: \mathcal{O}_{m}\left(x_{1}\right):: \mathcal{O}_{m}:\right]>-\left(R_{2} \leftrightarrow R_{1}\right)
\end{aligned}
$$

where $\mathcal{O}_{m}$ and $\Phi_{2}^{c} \equiv\left(\bar{\psi} \Gamma^{c} \psi\right)\left(\bar{\psi} \Gamma^{c} \psi\right)$ are the marginal scalar operators of the theory $\left(\Gamma^{c}=\left(1, \gamma_{\mu}, \sigma_{3}\right)\right)$. Notice that lower indices of Wilson coefficients have not been reported.

We consider this example very instructive because the values of the (differentiated) zero mass Wilson coefficients $C^{\mathcal{O}_{m}}, C^{\Phi_{2}^{c}}, \partial_{m} C^{\mathcal{O}_{m}}$ will be fixed imposing cancellation of I.R. divergences (of course the first two can be obtained in independent way from the massless theory).

In principle we should give proper normalization conditions for the one and two insertions of the operator $\mathcal{O}_{m} \equiv \bar{\psi} \psi$ in such a way that the Action Principle (Eq.4.6) with $k=1$ and $\Phi_{a}=\mathcal{O}_{m}$ ) holds. The point is that Action Principle is automatically satisfied in the massive theory if the usual MS definition for composite operators is adopted (see e.g. [32]). If we use for a moment the knowledge of the massive theory propagator, we obtain after standard calculations:

$$
\begin{aligned}
& <\left[: \mathcal{O}_{m}(r):: \mathcal{O}_{m}(0):\right]>_{m}=\int \frac{d^{2} p}{(2 \pi)^{2}} e^{-i p r} F(p, m) \\
& F(p, m) \equiv-\frac{1}{2 \pi} \int_{0}^{1} d x\left(\gamma_{E}+3+\log \left(\frac{m^{2}+x(1-x) p^{2}}{4 \pi \mu^{2}}\right)\right) \\
& <\left[\mathcal{O}_{m}\right]>_{m}=-\frac{m}{2 \pi}\left(\gamma_{E}+1+\log \left(\frac{m^{2}}{4 \pi \mu^{2}}\right)\right)
\end{aligned}
$$

It is easy to check that

$$
\partial_{m}<\left[\mathcal{O}_{m}\right]>_{m}=\int d^{2} r<\left[: \mathcal{O}_{m}(r):: \mathcal{O}_{m}(0):\right]>_{m}=F(0, m),
$$


i.e. Action Principle holds in this case without addition of contact terms.

Having we used only the knowledge of the massless theory, we would get Eq.(5.4) with

$$
F(p) \equiv F(p, 0)=-\frac{1}{2 \pi}\left(\gamma_{E}+1+\log \left(\frac{p^{2}}{4 \pi \mu^{2}}\right)\right) .
$$

We could also get a partial information on $<\left[\mathcal{O}_{m}\right]>_{m}$ imposing that the $\mu$ dependence (i.e. the U.V. behavior or in other words the anomalous dimensions) is the same in massive and massless theory:

$$
\mu \partial_{\mu} \partial_{m}<\left[\mathcal{O}_{m}\right]>_{m}=\mu \partial_{\mu} \int^{R} d^{2} x<\left[: \mathcal{O}_{m}(x):: \mathcal{O}_{m}(0):\right]>
$$

(notice the $m$ dependence of left side). The cutoff integral can be computed exactly by using the properties of Bessel functions [37:

$$
\begin{aligned}
& \int^{R} d^{2} x<\left[: \mathcal{O}_{m}(x):: \mathcal{O}_{m}(0):\right]>=\int \frac{d^{2} p}{(2 \pi)^{2}} F(p) \frac{2 \pi R}{p} J_{1}(p R) \\
& \left.=\frac{1}{2 \pi}\left(\log \left(\pi \mu^{2} R^{2}\right)+\gamma_{E}-1\right)\right)
\end{aligned}
$$

Integrating the differential equation (5.8) and imposing the dependence on $m / \mu$ we get

$$
\partial_{m}<\left[\mathcal{O}_{m}\right]>_{m}=-\frac{1}{\pi} \log \frac{m}{\mu}+\text { const }
$$

from which, integrating again, we get

$$
<\left[\mathcal{O}_{m}\right]>_{m}=-\frac{1}{\pi} m\left(\log \frac{m}{\mu}+\mathcal{C}_{\mathcal{O}_{m}}\right) .
$$

The constant $\mathcal{C}_{\mathcal{O}_{m}}$ can be fixed only imposing the Action Principle on the deformed theory (this gives the correct V.E.V., Eq. (5.5)) and its existence has been anticipated in Section 1 (notice that the two point correlator has zero I.R. degree of divergence and we expected I.R. problems in this case).

We computed some of the required integrals passing to complex coordinates $r=r^{1}+i r^{2} \cdots$ and using Stokes' Theorem to reduce surface integrals to line integrals. In Appendix A we give an explicit example of such calculations

The results (up to not relevant subleadings terms in the limit $R_{2}>>$ $\left.R_{1}>>|r|\right)$ are:

$$
\int^{R_{1}} d^{2} x_{1}<\left[: \mathcal{O}_{m}\left(x_{1}\right): \psi(r) \bar{\psi}(0)\right]>\sim-\frac{1}{2 \pi} \log \frac{R_{1}}{|r|}
$$




$$
\begin{gathered}
\int^{R_{1}} \int^{R_{2}}<\left[: \mathcal{O}_{m}\left(x_{1}\right):: \mathcal{O}_{m}\left(x_{2}\right):\left(\psi(r) \bar{\psi}(0)-C^{1}(r)\right)\right]>\sim \\
-\frac{\hat{r}}{8 \pi}\left\{\log \frac{|r|^{2}}{R_{1}^{2}}+\log \frac{|r|^{2}}{R_{2}^{2}}-2\right\}
\end{gathered}
$$

For what concerns the remaining integrals, it suffices to note that

$$
\int^{R_{1}} \int^{R_{2}}<\left[: \mathcal{O}_{m}:\left(x_{1}\right): \mathcal{O}_{m}:\left(x_{2}\right) \Phi_{2}^{a}(0)\right]>\propto \log \mu R_{1} \log \mu R_{2}+\cdots
$$

as it is easy to check.

Equating terms of same behavior in $R$, by Eq.(5.9), Eq.(5.13), Eq.(5.12), we get from Eq. (5.2)

$$
C^{\mathcal{O}_{m}}=-1 / 2 \quad \partial_{m} C^{1}=\frac{1}{4 \pi}\left(\log \left(\pi \mu^{2}|r|^{2}\right)+\gamma_{E}-1\right)
$$

and from Eq. (5.3)

$$
\begin{aligned}
& \partial_{m} C^{\mathcal{O}_{m}}=\frac{1}{4} \hat{r} \quad C^{\Phi_{2}^{a}}=0 \\
& \partial_{m}^{2} C^{1}=-\frac{1}{4 \pi}\left(\log \pi \mu^{2}|r|^{2}-2+\gamma_{E}\right) \hat{r} .
\end{aligned}
$$

Combining these results with the VEV of $\mathcal{O}_{m}$ Eq.(5.11) (and with the off critical information (5.5), we get

$$
\begin{aligned}
<\psi(r) \bar{\psi}(0)>_{m} \sim & -\frac{1}{2 \pi} \frac{\hat{r}}{|r|^{2}}\left(1+\frac{1}{2} m^{2}|r|^{2}\left(\log \left(\frac{m|r| e^{\gamma_{E}}}{2}\right)-\frac{1}{2}\right)\right) \\
& +\frac{m}{2 \pi} \log \left(\frac{m|r| e^{\gamma_{E}}}{2}\right)+O\left(m^{3}\right)
\end{aligned}
$$

that is the correct result as can be checked from the properties of $K$ functions. Another check can be obtained from the constraints

$$
\begin{aligned}
& \mu \frac{d}{d \mu} \partial_{m}^{n} C^{1}+n \partial_{m}^{n-1} C^{\mathcal{O}_{m}} \mu \frac{d}{d \mu}<\left[\mathcal{O}_{m}\right]>_{m}=0 \\
& \mu \frac{d}{d \mu} \partial_{m}^{n} C^{\mathcal{O}_{m}}=0
\end{aligned}
$$

obtained from the free theory relation

$$
0=\mu \frac{d}{d \mu}<\psi \bar{\psi}>_{m}=\mu \frac{d}{d \mu}\left(C^{1}(m)+C^{\mathcal{O}_{m}}(m)<\mathcal{O}_{m}>_{m}\right)
$$

equating terms of same order in $m$ and $\log m$. 


\subsection{Application to Ising Model}

We want to reconstruct here the mass corrections to short distance behavior of the two points spin-spin correlation function of the Ising Model by using the previously introduced method to compute Wilson coefficients. Similar results have been already obtained in a previous article [10]; we only want to treat this model as a concrete example of our reformulation to be compared with the preexisting approach. To put in evidence the renormalization scheme independence of our treatment (provided Hypotheses 1-3 are satisfied), we will use here the Hadamard finite part (see Appendix B) to deal with U.V. divergence and the I.R. regulator $\Theta(x)=J_{0}(|Q||x|)$ (see Eq. (3.12)) that is equivalent to insert $\Theta(x)=e^{i Q x}$ and then average over directions of $Q_{\mu}$ before taking the limit $Q \rightarrow 0$.

It is a well known fact [3] that the Ising model in proximity of its critical point is described by a theory of free Majorana fermions with mass $m$ and euclidean action

$$
S=\frac{1}{2 \pi} \int d^{2} x \psi \bar{\partial} \psi+\bar{\psi} \partial \bar{\psi}+i m \bar{\psi} \psi
$$

Moreover the exact correlator [34] is known from the scaling limit of the lattice theory and can be used as a check.

The conformal theory at fixed point $m=0$ is described by the primary operators $1, \sigma, \mathcal{E} \equiv: \frac{i}{2 \pi} \bar{\psi} \psi$ : of dimension $x=0,1 / 8,1$ from which the secondary operators can be built up by repeated application of the Virasoro generators:

$$
\Phi^{\{n\}\{\bar{n}\}}=L_{-n_{1}} \cdots L_{-n_{N}} \bar{L}_{-\bar{n}_{1}} \cdots \bar{L}_{-\bar{n}_{M}} \Phi,
$$

where $\Phi$ is any of the primary operators and the dimension of the secondary operator is $x=x_{\phi}+\sum n_{i}+\sum \bar{n}_{i}$. Away from the fixed point the expression of the operator conjugate to $\partial_{m}$ will be of the form $\left[\mathcal{O}_{m}\right]=-\mathcal{E}+<\left[\mathcal{O}_{m}\right]>1$.

At first order we have

$$
\partial_{m} C_{\sigma \sigma}^{1}(r)=\lim _{Q \rightarrow 0} \int d^{2} x e^{i Q x}<\left[-\mathcal{E}(x)\left(\sigma(r) \sigma(0)-C_{\sigma \sigma}^{\mathcal{E}}(r) \mathcal{E}(0)\right)\right]>
$$

(the average over directions of $Q_{\mu}$ to be taken after integrations is not reported for simplicity of notations).

Before proceeding to the computation of the integrals a precise definition of the operator $\mathcal{O}_{m}$ should be given in order to obtain well defined distributions from its insertions, as discussed in Section 4 . We define the double insertion of $\mathcal{O}_{m}$ by

$$
<\left[\mathcal{O}_{m}(r) \mathcal{O}_{m}(0)\right]>_{m}=\operatorname{Fp}_{\mu} \frac{m^{2}}{4 \pi^{2}}\left(K_{1}^{2}(m r)-K_{0}^{2}(m r)\right)
$$


where $\mathrm{Fp}_{\mu}$ means the distribution obtained taking the Hadamard finite part of the U.V. singularity. (We used some additional knowledge of the massive theory: the massless correlator can be derived by taking the limit $m \rightarrow 0$ of previous expression).

Proceeding as in Section 4 and imposing the Action Principle

$$
\partial_{m}<\left[\mathcal{O}_{m}\right]>_{m}=\int d^{2} r<\left[\mathcal{O}_{m}(r) \mathcal{O}_{m}(0)\right]>_{m}=-\frac{1}{2 \pi}\left(\log \left(\frac{m}{2 \mu}\right)+1-\psi(1)\right)
$$

(see $(\overline{B .6})$ for computation of the integral), we derive the V.E.V.:

$$
<\left[\mathcal{O}_{m}\right]>_{m}=-\frac{m}{2 \pi} \log \left(\frac{m e^{\gamma_{E}}}{2 \mu}\right) .
$$

We recall that (as explained in Section 4 and in Section 5.1) the numerical constant inside the logarithmic term cannot be fixed only by knowledge of the $m=0$ theory.

We can now come back to the computation of integrals in Eq.(5.21).

The (locally integrable) correlation functions involved in the first integral is exactly known from the conformal theory [33]:

$$
<\mathcal{E}\left(r_{1}\right) \sigma(r) \sigma(0)>=\frac{r^{3 / 4}}{4 \pi\left|r_{1}-r\right|\left|r_{1}\right|}
$$

and the integral can be computed (in the limit $Q \rightarrow 0$ ) by standard Feynman diagram techniques and properties of Bessel Functions [37]:

$$
\begin{aligned}
& \int d^{2} x \frac{1}{|x-r|} \frac{1}{|r|} e^{i Q x}=\int_{0}^{1} d t \frac{e^{i(Q r) t}}{(t(1-t))^{1 / 2}} \int_{0}^{\infty} \frac{d s}{s} e^{-s} e^{-t(1-t) Q^{2} r^{2} /(4 s)} \\
& =2 \int_{0}^{1} d t \frac{e^{i(Q r) t}}{(t(1-t))^{1 / 2}} K_{0}(\sqrt{t(1-t)}|Q \| r|) \sim-2 \pi \log \frac{|Q \| r| e^{\gamma_{E}}}{8}
\end{aligned}
$$

For what concerns the second integral in (5.21) we have (after the limit $m \rightarrow 0$ is taken)

$$
\begin{aligned}
\int d^{2} x e^{i Q x}<[\mathcal{E}(x) \mathcal{E}(0)]> & =\int d^{2} x e^{i Q x} \mathrm{Fp}_{\mu} \frac{1}{4 \pi^{2} x^{2}} \\
& =\frac{-1}{2 \pi}\left(\log \frac{|Q|}{2 \mu}+\gamma_{E}\right)
\end{aligned}
$$

(see [35] for the Fourier transform of Hadamard finite part). Substituting (5.26)-(5.27) in Eq.(5.21) we get

$$
\partial_{m} C_{\sigma \sigma}^{1}=\frac{1}{2} r^{3 / 4}\left(\log \frac{|Q||r|}{2}+\gamma_{E}-\log 4\right)-C_{\sigma \sigma}^{\mathcal{E}} \frac{1}{2 \pi}\left(\log \frac{|Q|}{2 \mu}+\gamma_{E}\right) .
$$


Cancellation of the $\log Q$ factor fixes

$$
C_{\sigma \sigma}^{\mathcal{E}}(r)=\pi r^{3 / 4}
$$

while equating the regular term we get

$$
\partial_{m} C_{\sigma \sigma}^{1}=r^{3 / 4} \frac{1}{2} \log \left(\frac{\mu r}{4}\right)
$$

From (5.30) we can thus reconstruct at first order in $m$ the deviation from the scaling behavior of the fixed point theory for the Wilson coefficient

$$
C_{\sigma \sigma}^{1}(r, m ; \mu)=\frac{1}{r^{1 / 4}}\left(1+\frac{m r}{2} \log \left(\frac{\mu r}{4}\right)+O\left((m r)^{2}\right)\right)
$$

and (using also (5.29) ) of the short distance behavior of the two point function:

$$
\begin{aligned}
<\sigma(r) \sigma(0)>_{m} & \sim C_{\sigma \sigma}^{1}(r, m ; \mu)+m \partial_{m} C_{\sigma \sigma}^{m}(r, m=0)<\mathcal{O}_{m}>_{m} \\
& =\frac{1}{r^{1 / 4}}\left(1+\frac{m r}{2} \log \left(\frac{m r}{8} e^{\gamma_{E}}\right)+O\left((m r)^{2}\right)\right) .
\end{aligned}
$$

These results are in agreement with those of [10] and with the exact ones of 34.

\section{Conclusions}

We presented a consistent method to deal with I.R. singularities of expansions in a relevant parameter around a field theory without super-renormalized couplings. Our approach is a reformulation and an extension of other recent works [8, 9, 10]. The goal is the reconstruction of the short distance behavior (Wilson coefficients) of the deformed theory obtained by adding a relevant operator to the action. The use of Action Principle and (weak) convergence property of O.P.E. give closed and I.R. finite expressions for the derivatives with respect to generalized masses of Wilson coefficients that, can thus be reconstructed by means of Taylor expansion (that is convergent if analyticity of Wilson coefficients can be assumed, otherwise asymptotic).

Our contribution is an orthodox treatment of U.V. divergences of composite operators that, as a consequence, allows for an explicit and simple inductive proof of I.R. finitess and of general equation Eq.(3.12). We put in evidence that Eq.(3.12) does not refer to a particular U.V. renormalization scheme (provided Hyp.1-3 are satisfied) and holds for general I.R. regulators. 
We discussed also the presence of well defined but unknown constants related to validity of renormalized Action Principle that parameterize the long distance behavior of deformed theory (essentially operators' V.E.V.) and cannot be computed by using the knowledge of the massless theory only. Nevertheless these constant are universally present in short distance expansions of different products of composite operators and are always in finite number at any finite order in the masses. The possibility of fixing these parameters (e.g. by some symmetry relation between operators in the deformed theory or the knowledge of some particular deformed correlator, as in the Ising model of Section 5.2) depends on particular models and should be discussed case by case.

In spite of this problem, we think that its simplicity and generality, compared to many specific approaches, make of this method a powerful and promising tool.

We remind in particular that the (three) main hypotheses on which it relies have been found to be satisfied for the bulk of the interesting conformal field theories if adequate renormalization schemes are used: it thus seem that the technique could be helpful in the investigation of relevant deformations of those theories. We hope to come back to the problem soon.

Acknowledgements: The authors are grateful to C.M. Becchi for suggesting to them the problem and for many useful discussions. We acknowledge also for interesting discussions R. Collina, G. Delfino, K. Konishi, C. Imbimbo, Hiroshi Suzuki, J.-B. Zuber. Part of the work of one author (R.G.) was realized during visits to S.Ph.T. of Saclay and C.E.R.N., whose staffs are acknowledged for the warm hospitality.

\section{Appendix A Complex integrals}

We give here an example of the computations required to obtain Eqs.(5.23) the detailed calculation of the integral

$$
I \equiv \int_{|z| \leq R} d^{2} z \frac{1}{(z-|r|)^{*}} \frac{1}{z-z^{\prime}}
$$

in which $z, z^{\prime}, r$ are complex variables $\left(z^{\prime} \neq|r|\right)$ and $d^{2} z \equiv \frac{i}{2} d z \wedge d z^{*}$. A trick to simplify things is to use Stokes' theorem: given a one form $\Omega$ regular in the domain $\Sigma$ one has

$$
\int_{\Sigma} d \Omega=\int_{\partial \Sigma} \Omega
$$


If we pose $\Omega=\omega\left(z, z^{*}\right) d z+\bar{\omega}\left(z, z^{*}\right) d z^{*}$ we can fix $\Omega$ in our case requiring:

$$
\left(\frac{\partial \bar{\omega}}{\partial z}-\frac{\partial \omega}{\partial z^{*}}\right)=\frac{i}{2} \frac{1}{(z-|r|)^{*}} \frac{1}{z-z^{\prime}} .
$$

With the choice $\bar{\omega}=0$ and $\omega=-\frac{i}{2} \log \left(z^{*}-|r|\right) /\left(z-z^{\prime}\right)$ we get

$$
I=-\frac{i}{2} \int_{\gamma} \log \left(z^{*}-|r|\right) /\left(z-z^{\prime}\right) d z
$$

in which the path $\gamma$ should not enclose singularities (pole in 0 and cut from $|r|$ that we choose along the real positive axis) to allow for application of Stokes' Theorem. We split $\gamma=\gamma_{1} \cup \gamma_{2} \cup \gamma_{3} \cup \gamma_{4}$ where (in terms of z):

$$
\begin{aligned}
& \gamma_{1} \equiv\left\{z / z=z^{\prime}+\epsilon e^{i \phi}, \phi \in(2 \pi, 0)\right\} \\
& \gamma_{2} \equiv\left\{z / z=\rho \in[|r|, R] \text { or } z=e^{2 \pi i} \rho, \rho \in(R,|r|)\right\} \\
& \gamma_{3} \equiv\left\{z / z=\operatorname{Re}^{i \phi}, \phi \in(0,2 \pi)\right\}
\end{aligned}
$$

(notice the orientations of the intervals). The contribution of the path $\gamma_{4}$ turning around $|r|$ and connecting the two branches of $\gamma_{2}$ is easily seen to be zero.

Performing easy integrations we get the desired result:

$$
I \sim-\pi \log \left(\frac{\left|z^{\prime}-\right| r||^{2}}{R^{2}}\right)+O\left(\frac{|r|}{R}, \frac{\left|z^{\prime}\right|}{R}\right)
$$

All the integrals in Eqs. (5.23) can be computed at relevant order by (repeated) application of Stokes' Theorem.

\section{Appendix B Computation of the Hadamard finite part}

In one dimension Hadamard finite part 355 of a non locally summable (in $x=0$ ) function

$$
g(x)=\sum_{\nu} \frac{A_{\nu}}{x^{\lambda_{\nu}}}+\frac{A_{1}}{x}+h(x)
$$

(in which $\lambda_{\nu}$ is a finite set of complex indices with $\operatorname{Re} \lambda_{\nu} \geq 1$ but different from 1 and $h(x)$ is regular) is

$$
F p \int_{0}^{b} g(x) d x \equiv \lim _{\epsilon \rightarrow 0}\left\{\int_{\epsilon}^{b} g(x)-I(\epsilon)\right\}
$$


in which the infinite part is defined as

$$
I(\epsilon) \equiv \sum_{\nu} \frac{A_{\nu}}{\lambda_{\nu}-1}\left(\frac{1}{\epsilon}\right)^{\lambda_{\nu}-1}+A_{1} \log \frac{1}{\epsilon} .
$$

In this case we obtain

$$
F p \int_{0}^{b} g(x) d x=-\sum_{\nu} \frac{A_{\nu}}{\lambda_{\nu}-1}\left(\frac{1}{b}\right)^{\lambda_{\nu}-1}-A_{1} \log \frac{1}{b}+\int_{0}^{b} h(x) d x .
$$

One can extend a locally non summable function as a tempered distribution by taking the Hadamard finite part of the integral of the function times the test function. For our purposes we are involved only in radially symmetric functions and this case is a trivial generalization of one dimensional situation. Extension to higher number of dimensions can be found in [35] and [36]. In particular in [36] is given a detailed treatement of distributional extensions of homogeneous functions of many variables that could be useful in the treatment of conformal field theories singularities. Notice also that Hadamard finite part, subtracting only divergent part is a Minimal Subtraction in the sense explained in Section 2.

We compute now the integral

$$
I=\int d^{2} r \mathrm{Fp}_{\mu} \frac{m^{2}}{4 \pi^{2}}\left(K_{1}^{2}(m r)-K_{0}^{2}(m r)\right)
$$

The integral of this distribution is easily done by taking the finite part at $\mu r=$ 0 i.e. by subtracting the divergent part in $\mu r=0$ (we added a renormalization point scale $\mu$ to keep correct dimensions):

$$
\begin{aligned}
& \mathrm{Fp}_{\mu} \int_{\epsilon \leq|r|} d^{2} r \frac{m^{2}}{4 \pi^{2}}\left(K_{1}^{2}(m r)-K_{0}^{2}(m r)\right)=\frac{1}{2 \pi} \mathrm{Fp}_{\mu} \int_{m \epsilon}^{\infty} d x x\left(K_{1}^{2}(x)-K_{0}^{2}(x)\right) \\
& =\frac{1}{2 \pi} \mathrm{Fp}_{\mu}\left(\frac{x^{2}}{2}\left(2 K_{1}^{2}(x)-K_{0}(x)^{2}-K_{0}(x) K_{2}(x)\right)\right)_{m \epsilon}^{\infty} \\
& =-\frac{1}{2 \pi} \mathrm{Fp}_{\mu}\left(\log \left(\frac{m \epsilon}{2}\right)+1-\psi(1)\right) \\
& =-\frac{1}{2 \pi}\left(\log \left(\frac{m}{2 \mu}\right)+1-\psi(1)\right)
\end{aligned}
$$

where the properties of the $K_{\nu}(z)$ functions (in particular their behavior in $z=0$ and in $z=\infty$ ) have been used, see for instance [37].

\section{References}


[1] R. Jackiw and S. Templeton, Phys.Rev. D23 (1981) 2291.

M.C. Bergére and F. David, Annals of Phys. 142 (1982) 416.

[2] H. Saleur and C.Itzykson, Journ. of Stat. Phys. 48 (1987) 449.

Vl.S.Dotsenko, Nucl. Phys. B314 (1989) 687.

A. Cappelli and J.I. Latorre Nucl.Phys. B340 (1990) 659.

[3] A.A. Belavin, A.M. Polyakov and A.B. Zamolodchikov, Nucl. Phys. B241(1984)333.

[4] C.Itzykson, H.Saleur and J.-B. Zuber, eds., "Conformal invariance and applications to Statistical Machanics", World Scientific 1988.

[5] A.B. Zamolodchikov, Sov.Journ. Nucl.Phys. 46 (1987) 1090.

A.A. Ludwig and J.L. Cardy Nucl.Phys. B285 (1987) 687.

[6] A.B. Zamolodchikov and Al.B. Zamolodchikov, Annals of Phys. 120 (1979) 253.

[7] J. L. Cardy in "Fields, Strings and Critical Phenomena", Les Houches Session XLIX edited by E. Brezin and J. Zinn-Justin, Elsavier, 1989.

A. B. Zamolodchikov, Reviews in Math. Phys. 1 (1990) 197.

[8] Al.B. Zamolodchikov, Nucl.Phys. B 348 (1991) 619

[9] H.Sonoda, Nucl.Phys. B383 (1992) 172; Nucl.Phys. B394 (1993) 302.

[10] B. Mikhak and A.M. Zarkesh, Nucl.Phys. B430 [FS](1994) 656.

[11] K.G. Wilson, Phys.Rev. 179 (1969) 1499.

[12] F. Wegner, J.Phys. A8 (1975) 710.

[13] H.Sonoda, Nucl.Phys. B352 (1991) 585; Nucl.Phys. B352 (1991) 601.

[14] J. Schwinger, Phys. Rev. 82 (1951) 914; Phys. Rev. 91 (1953) 713.

[15] J.H. Lowenstein, Comm. Math. Phys. 24 (1971) 1.

Y.M.P. Lam, Phys. Rev. D6 (1972) 2145; Phys. Rev. D7 (1973) 2943.

T.E. Clark and J.H. Lowenstein, Nucl.Phys. B113 (1976) 109.

[16] P. Breitenlohner and D.Maison, Comm. Math. Phys. 52 (1977) 11,39,55.

M.C. Bergére and Y.M.P. Lam, J. Math. Phys. 17 (1976) 1546. 
[17] E.R. Speer, "Dimensional and analytic renormalization" in "Renormalization theory", G. Velo and A.S. Wightman eds., D. Reidel Pub. Co., 1976.

[18] V.A. Smirnov, Z. Phys. C 67 (1995) 531.

[19] G. t'Hooft and M. Veltman, Nucl. Phys. B44 (1972) 189.

J. C. Collins, Nucl. Phys. B80 (1974) 341.

[20] S.G. Gorishny, S.A. Larin and F.V. Tkachov, Phys.Lett.124B (1983) 339.

C.H. LLewellyn Smith and J.P. de Vries, Nucl. Phys. B296 (1988) 991.

[21] A.M. Polyakov, Sov. Phys. JETP 39 (1974) 10.

G. Mack Nucl. Phys. B118 (1977) 445.

[22] G. Mack, Comm. Math. Phys. 53 (1977) 155.

[23] K.G. Wilson and W. Zimmermann, Comm. Math. Phys. 24 (1972) 87.

[24] W. Zimmermann, Annals of Phys. 77 (1973) 570.

T.E. Clark, Nucl. Phys. B81 (1974) 263.

P.S. Collecott, Annals of Phys. 113 (1978) 461.

[25] N.N. Bogoliubov and O.V. Shirkov, "Introduction to the Theory of Quantized Fields", 4th Ed. Wiley, NY, 1980.

K. Hepp, Comm. Math. Phys. 2 (1966) 301.

H. Epstein and V. Glaser, Ann. Inst. Poinc. XIX (1973) 211.

[26] C.M. Becchi, in "Fields and Particles", Proceedings of the XXIX Int. Universitästwochen fur kernphysik, Schladming, Austria 1990, Eds. Mitter and Schweiger, Springer Verlag Berlin Heidelberg 1991.

[27] P.S. Collecott, Nucl. Phys. B135 (1978) 167.

[28] J. Zinn-Justin, Quantum Field Theory and Critical Phenomena, Claredon Press, Oxford 1989.

[29] G.M. Shore, Nucl. Phys. B362 (1991) 85.

[30] G.t'Hooft, Acta Physica Austriaca, XXII (1980) 531, Springer-Verlag 1980. 
[31] D. Kutasov, Phys. Lett. B220 (1989) 153.

M. Lässig, Nucl. Phys. B334 (1990) 647.

H.Sonoda, "Connections on the theory space", hep-th/9306119.

B.P. Dolan, Int. J. Mod. Phys. A10 (1995) 2439.

[32] J.C. Collins, "Renormalization", Cambridge Univ. Press, 1984.

[33] P. Di Francesco, H. Saleur, and J.-B. Zuber, Nucl. Phys. B290 (1987) 527.

[34] T.T. Wu, B.M. McCoy, C.A.Tracy and E.Barouch, Phys. Rev. B13 (1976) 316.

[35] L. Schwartz, "Théorie des distributions", Tome I, Hermann Paris, 1966.

[36] I.M. Gelfand, G.E. Shilow, "Generalized Functions", Vol. I, Academic Press, 1964.

[37] I.S. Gradshtein and I.M. Ryzhik, "Tables of Integrals, Series and Products", Academic Press. 